\title{
Design and protocols of the GIRAF prevention trial: A randomized, prospective, parallel, two-center study to compare cognitive outcomes in subjects with atrial fibrillation using warfarin or dabigatran
}

\author{
André A. S. F. de Toledo ${ }^{1}$, Pai C. Yu ${ }^{2}$, Danielle M. Guallandro ${ }^{2}$, Daniela Calderaro ${ }^{2}$, \\ Gabriel A. L. do Carmo ${ }^{1}$, Breno F. S. Fernandes ${ }^{1}$, Marina A. Neves ${ }^{1}$, Raul Feitosa ${ }^{2}$, \\ Ana Carolina Rodrigues ${ }^{1}$, Antônio Luiz P. Ribeiro ${ }^{1}$, Ricardo Nitrini ${ }^{2}$, Paulo Caramelli ${ }^{1}$, \\ Bruno Caramelli ${ }^{2} *$
}

\author{
${ }^{1}$ Federal University of Minas Gerais, Belo Horizonte, MG, Brazil \\ ${ }^{2}$ University of São Paulo Medical School, São Paulo, SP, Brazil
}

Received: 25 October 2015

Accepted: 18 November 2015

\section{*Correspondence: \\ Bruno Caramelli, \\ E-mail: bcaramel@usp.br}

Copyright: (c) the author(s), publisher and licensee Medip Academy. This is an open-access article distributed under the terms of the Creative Commons Attribution Non-Commercial License, which permits unrestricted non-commercial use, distribution, and reproduction in any medium, provided the original work is properly cited.

\section{ABSTRACT}

Background: Atrial fibrillation (AF) is the most common cardiac arrhythmia and is associated with cognitive impairment and dementia. Cognitive and functional impairments have been neglected in clinical trials with patients affected by AF. Dabigatran is a new drug, which can offer a more stable long-term anticoagulant therapy compared to warfarin. This advantage could be associated with a minor incidence of cognitive and functional decline. The objective of the study is to evaluate the effects of dabigatran compared with warfarin on cognitive and functional impairments, thrombin production, bleeding occurrence and cerebrovascular complications in elderly patients with AF.

Methods: A randomized, prospective parallel, two-center, active-controlled trial will be performed in a sample of 200 patients with AF randomly designated to take dabigatran (150 mg twice daily) or warfarin (once daily, dose controlled by INR between 2 and 3). After one and two years, subjects will be assessed considering cognitive outcomes. Additionally, patients will be submitted to brain magnetic resonance imaging (MRI) at the beginning and ending of study to identify possible cerebrovascular events, as well as carotid ultrasonography to search for atherosclerotic disease.

Conclusions: The GIRAF study expects to provide data about prevention of cognitive and functional impairments among patients on anticoagulant therapy for AF, and to explore new concepts on potential prevention of cognitive decline and benefits for AF patients and their relatives.

Clinical Trial Registration: ClinicalTrials.gov - Unique identifier: NCT01994265, registered on November 9, 2013

Keywords: Atrial fibrillation, Anticoagulant therapy, Cognitive impairment, Dementia, Warfarin, Dabigatran

\section{INTRODUCTION}

Atrial fibrillation (AF) is the most common cardiac arrhythmia and its incidence increases with aging. Cerebrovascular complications represent a major challenge and have been intensively assessed in clinical trials with patients affected by AF. It is known that prevention of ischemic stroke is the most common and studied outcome in trials with AF patients. Conversely, cognitive and functional impairments have been partially 
neglected in these studies. A recent post-hoc analysis studied 31,546 patients with atrial fibrillation and concluded that even in absence of stroke, cognitive and functional impairments were associated with AF in longterm follow-up. ${ }^{1}$ Another recent meta-analysis concluded that atrial fibrillation is independently associated with increased risk of dementia. ${ }^{2}$ In the present scenario, in which global aging is a major challenge, not only quantity but also quality of life are objectives equally important, with prevention of cognitive and functional declines being of utmost relevance. Moreover, a community-based longitudinal cohort study with 5,888 participants found that in the absence of clinical stroke, patients with AF are more likely to have a diagnosis of cognitive impairment or dementia at earlier stages compared with people with no history of AF. ${ }^{3}$ Finally, a randomized controlled trial evaluating 973 patients, who were recruited from primary care and randomly assigned to warfarin or aspirin, found no evidence that warfarin confers clinically important protection over aspirin against cognitive decline in $\mathrm{AF}$ in the first 33 months of treatment. $^{4}$

The standard therapeutic regimen used to prevent ischemic neurovascular events related to $\mathrm{AF}$ is the anticoagulation therapy with warfarin. However, treatment with warfarin is related to some important issues such as the narrow therapeutic index, with toxic dose associated with severe bleeding events. This feature demands a rigorous control with periodic coagulation tests (prothrombin time). Furthermore, there are interactions with numerous drugs and types of food including alcohol. Probably because of that, patients with AF using warfarin are undertreated or stay for a long time outside the therapeutic index..$^{5-7}$ Nevertheless, warfarin has proven to be effective and safe for the prevention of neurovascular events in different subgroups of patients in the last 60 years. Recently, new anticoagulant drugs were developed and revealed to be non-inferior to warfarin for the prevention of stroke. These medications have additional advantages in relation to warfarin because they can offer a more stable anticoagulant therapy in longterm, besides the absence of interaction with food and alcohol. This major stability could be responsible for offering increased anticoagulation effectiveness for longer periods. However, this advantage would not be identified for greater outcomes such as stroke, but could be recognized for equally important outcomes such as cognitive and functional impairments not analysed so far.

\section{Objectives}

\section{Primary objective}

- To evaluate the effect of dabigatran in comparison to warfarin considering cognitive and functional impairments as results of the disease itself or its progression in elderly patients with AF.

\section{Secondary objectives}

- To evaluate the effect of dabigatran in comparison to warfarin considering the production of thrombin in elderly patients with AF.

- To evaluate the effect of dabigatran in comparison to warfarin considering the occurrence of major bleedings in elderly patients with AF.

- To evaluate the effect of dabigatran in comparison to warfarin considering the prevention of transitory ischemic attack (TIA) or stroke in elderly patients with AF.

\section{METHODS}

\section{Study design}

The GIRAF Prevention Trial (acronym for CoGnitive Impairment Related to Atrial Fibrillation) is a randomized, prospective, parallel clinical trial, blinded in what it concerns to interpretation of outcomes (Figure 1). It will include 200 patients in two centers from Brazil University of São Paulo Medical School (coordinating centre) and Federal University of Minas Gerais. It is estimated to last 36 months considering final data collection date for primary outcome measurement.



Figure 1: Symbol of the GIRAF prevention trial.

Cognitive and functional impairments observed in patients with atrial fibrillation are related to thrombotic or cardio embolic events. Concerning cognitive and functional impairments outcomes, our hypothesis is that warfarin use is less efficient than dabigatran, an anticoagulant with more stable action profile. Consequently, dabigatran could offer protection against thrombotic events and therefore attenuate the process of cognitive and functional decline associated with AF.

The study was approved by institutional review boards from both participating centers and all participants will provide written informed consent before entering the trial. 


\section{Sample size}

Based on a previous study, which assessed patients with AF and analysed the potential benefits of dabigatran for its increased therapeutic stability, the estimated occurrence rates of cognitive outcome were $20 \%$ and $40 \%$ in subgroups of dabigatran and warfarin, respectively. ${ }^{1}$ Pondering a bicaudate test with $80 \%$ of power and $\alpha$ equals to 0.05 , the necessary number of patients would be 91 per group. Finally, considering possible dropouts, the final sample size consists on 100 patients in each treatment group.

\section{Inclusion criteria}

1. Confirmed and registered diagnosis of atrial fibrillation (AF) or atrial flutter by one of the following criteria:

A. Electrocardiography (ECG) in the first day of evaluation or randomization.

B. Patient presented symptomatic episode of paroxystic or persistent AF, registered by ECG within six months before randomization.

C. Documentation compatible with paroxystic or persistent AF, symptomatic or asymptomatic, in two different occasions separated by at least one day, provided that one of them occurred within six months before randomization. In this case, $\mathrm{AF}$ can have been registered by ECG, rhythm monitor or pacemark/implantable/defibrillator/Holter evaluation.

2. Age equal or superior to 70 years.

3. Men or women.

4. CHA2DS2-VASc Score > 1 (acronym for Congestive heart failure/Left ventricle dysfunction, Hypertension, Age $\geq 75$ years, Age 65-74 years, Diabetes mellitus, Sex category, Stroke/TIA/Thromboembolism, Vascular disease). ${ }^{8}$

\section{Exclusion criteria}

1. Valvular heart disease (prosthesis or clinically relevant disease).

2. Previous TIA or stroke.

3. Dementia or other severe neurological condition.

4. Severe psychiatric disease (bipolar disease, major depression, schizophrenia)

5. Major surgeries in the last 30 days.

6. Surgery plans for the following three months.

7. Intracranial, intra-ocular, spinal, retroperitoneal or intra-articular hemorrhage in absence of trauma.

8. Gastrointestinal bleeding in the last 12 months.

9. Symptomatic gastric ulcers or detected by endoscopy within the last 30 days.

10. Bleeding disease or diathesis.

11. Use of fibrinolytic agents in the last 48 hours.

12. Uncontrolled hypertension (systolic blood pressure > $180 \mathrm{mmHg}$ or diastolic blood pressure > 100 $\mathrm{mmHg})$.
13. Active cancer or radiotherapy in the last six months with life expectancy inferior to three years.

14. Contraindication to the use of warfarin.

15. Reversible or circumstantial causes of atrial fibrillation (e.g. post-operation, cardiac surgery, pulmonary embolism, hyperthyroidism).

16. Planed surgery for the treatment of atrial fibrillation (ablation).

17. Creatinine clearance inferior to $30 \mathrm{~mL} / \mathrm{min}$.

18. Active infectious endocarditis.

19. Active hepatic disease defined by hepatitis (A,B or $\mathrm{C}$ virus) or transaminases in levels greater than twofold the reference values.

20. Anemia (hemoglobin $<10.0 \mathrm{mg} \%$ ) or thrombocytopenia (platelets lower than 100,000/mm3).

21. Important ventricular failure defined by ejection fraction inferior to $35 \%$ at echocardiography.

22. Pacemaker, metallic devices (prosthesis or clips) or any condition that contraindicates cerebral magnetic resonance.

\section{Procedures and follow-up}

Patients will be randomized to take dabigatran $(150 \mathrm{mg}$ twice daily, $110 \mathrm{mg}$ twice daily for patients above 80 years or with creatinine clearance $(\mathrm{CrCl})$ between 30 and $50 \mathrm{~mL} / \mathrm{min}$ ) or warfarin (once daily, dose controlled by INR between 2 and 3) for two years. For patients already in use of oral anticoagulation a controlled washout will be performed before randomization as following:

1. For the conversion of warfarin to dabigatran, it will be initiated dabigatran when INR $<2$.

2. For the conversion of dabigatran (or other oral anticoagulation) to warfarin, warfarin treatment will be initiated according to $\mathrm{CrCl}$ :

A. $\mathrm{CrCl} \geq 50 \mathrm{~mL} / \mathrm{min}$, initiate warfarin three days before discontinuation of dabigatran.

B. $\mathrm{CrCl}$ between 30 and $50 \mathrm{~mL} / \mathrm{min}$, initiate warfarin two day before discontinuation of dabigatran.

After one year and at the end of study (two years), individuals will be assessed regarding cognitive endpoints following the National Institute of Neurological Disorders and Stroke-Canadian Stroke Network Vascular Cognitive Impairment Harmonization Standards. ${ }^{8}$ These standards recommend three evaluation protocols (five, 30 and 60-minutes) with several tests, highlighting the Mini-Mental State Examination (MMSE). ${ }^{9,10}$ In our study, investigators will use a neuropsychological tests list (See below) based on the 60minutes evaluation protocol, complemented by computerized neurophysiological tests and the Montreal Cognitive Assessment (MoCA). The MoCA is considered to have a sensitivity of $90 \%$ in identifying mild cognitive impairment (MCI) compared to a sensitivity of $18 \%$ with the MMSE. ${ }^{11,12}$ 
The main outcomes are the occurrence rates of cognitive and functional decline, including dementia, in both treatment groups, by the end of the study.

Neurologists will perform all the cognitive evaluations during medical consultations every six months. Furthermore, patients will be submitted to brain magnetic resonance imaging (MRI) in the beginning and ending of study to identify possible cerebrovascular events as well as carotid ultrasonography to search for atherosclerotic disease.

Inclusion and exclusion cardiologic criteria will be analyzed by cardiologists, who are going to follow patients during the whole period of study performing medical consultations every three months for clinical control.

As a randomized, prospective and blinded trial in what it concerns to interpretation of outcomes, objective cognition measurements performed by experienced neurologists can establish a reliable difference between warfarin and dabigatran in this outcome. Moreover, cardiologists will closely monitor every side effect, risk of overdose or other unpredictable issues during the whole study.

\section{Neuropsychological tests list}

\section{Global cognition}

Mini-mental state examination (MMSE) $)^{9,10}$

Montreal cognitive assessment (MoCA) $)^{11,12}$

\section{Executive/activation}

Animal naming (semantic fluency) ${ }^{8}$

Controlled oral word association test (COWAT) FAS $^{13,14}$

WAIS-III digit symbol-coding ${ }^{15}$

Trail-making test - Part B ${ }^{16}$

\section{Language/lexical retrieval}

Boston naming test (BNT), short form ${ }^{17}$

\section{Visuospatial}

Clock drawing test (CDT) ${ }^{18,19}$

\section{Memory/learning}

Figure memory test ${ }^{20,21}$

\section{Neuropsychiatric/depressive symptoms}

Neuropsychiatric inventory (NPI) 22,23

Geriatric depression scale (GDS), short form ${ }^{24,25}$

\section{Computerized neuropsychological tests}

Selective visual attention test

Divided visual attention test

Sustained visual attention test

Reaction time test

Other

Informant questionnaire on cognitive decline in the elderly (IQCODE), short form ${ }^{26,27}$

\section{Computerized neuropsychological Tests}

The Selective Visual Attention Test consists of 60-frames stimuli presented within two minutes. The subjects are requested to identify if a proposed target-stimulus is displayed on a near group of three stimuli. This test aims to assess the ability of targeting attention to a certain stimulus by measuring two variables - reaction time and accuracy.

The Divided Visual Attention Test involves the presentation of 60 words (substantives) in the middle of a screen with an alternating confounding stimulus (a figure of a star) within two minutes. The subjects are requested to identify which words start with letter "C" whilst they have to say the word "star" every time they see this figure-stimulus. Therefore, this test consists of two tasks and aims to assess the ability of divided attention between two visual stimuli.

The Sustained Visual Attention Test implicates the exhibition of a number ("1", " 2 " or " 3 ") in the middle of a screen, alternated by a different stimulus (symbols "+" and "=") with an increasing frequency, within four minutes. The subjects are requested to answer quickly if the numbers " 1 ", " 2 " or " 3 " are being shown in a course of 54 attempts. This test aims to assess the ability of visual attention maintenance during a certain period of time.

Finally, the Reaction Time Test is based on the presentation of a figure of a square in the middle of a screen during variable time intervals (between 500 and 2,500 seconds). During the test, the square is showed 40 times and the subjects are requested to react quickly at each occurrence of the figure.

Each described test will be preceded by standard instructions exhibited in the screen, concerning the task to be accomplished. Moreover, after presentation of instructions, subjects will receive a quick training to permit a better knowledge about the task to be performed.

\section{DISCUSSION}

The worldwide phenomenon of demographic transition over recent decades has produced an expressive increase in the number of elderly people. A significant 
consequence of this process is an expansion of chronicdegenerative conditions, especially dementia and cognitive disorders. ${ }^{28}$

A recent review about the relationship between $\mathrm{AF}$ and dementia stated increasing evidence that $\mathrm{AF}$ is associated with a higher risk of dementia. AF and dementia are conditions that mainly affect elderly patients. Moreover, both diseases share multiple common risk factors (e.g. diabetes mellitus, chronic kidney disease, vascular disease, heart failure, inactivity/low activity, genetics/inheritance, sleep apnea, hypertension, alcohol consumption), which may be prevention targets simultaneously. Likewise, it is suggested that in patients with $\mathrm{AF}$, choices regarding anticoagulation as well as rhythm and rate-control could play an important role in the risk/progression of dementia. ${ }^{29,30}$

It is recognized that vascular cognitive impairment, as observed in AF patients with stroke and TIA, is mainly associated with deficits in executive function, attention and information processing. ${ }^{31,32}$ Afterwards, it is clear that efforts to improve quality of life focused on patients with AF by preventing unfavorable outcomes such as cognitive and functional impairments are extremely important and necessary.

As can be inferred from another recent review about cognitive function in AF, our study is going to be the first of its kind to access the hypothesis that elderly patients with AF can benefit from dabigatran for prevention of cognitive and functional decline compared to warfarin. ${ }^{32}$

\section{CONCLUSION}

The GIRAF study intends to provide data about prevention of cognitive and functional impairments among patients in anticoagulant therapy for $\mathrm{AF}$, expecting to modify the standard therapeutic regimen and to explore new concepts of cognitive sparing and benefits for patients and their relatives.

\section{Funding: Boehringer Ingelheim International GMBH, Ingelheim, Germany \\ Conflict of interest: None declared \\ Ethical approval: The study was approved by institutional review boards from both participating centres (University of São Paulo Medical School and Federal University of Minas Gerais).}

\section{REFERENCES}

1. Marzona I, O'Donnell M, Teo K, Gao P, Anderson $\mathrm{C}$, Bosch $\mathrm{J}$ et al. Increased risk of cognitive and functional decline in patients with atrial fibrillation: results of the ONTARGET and TRANSCEND studies. Can Med Assoc J. 2012;184(6):E329-36.

2. Santangeli P, Di Biase L, Bai R, Mohanty S, Pump $\mathrm{A}$, Brantes $\mathrm{MC}$ et al. Atrial fibrillation and the risk of incident dementia: a meta-analysis. Heart Rhythm. 2012;9(11):1762-8.

3. Thacker EL, McKnight B, Psaty BM, Longstreth WT, Sitlani CM, Dublin S, et al. Atrial fibrillation and cognitive decline - A longitudinal cohort study. Neurology. 2013;81:119-25.

4. Mavaddat N, Roalfe A, Fletcher K, Lip GYH, Hobbs R, Fitzmaurice D et al. Warfarin Versus Aspirin for Prevention of Cognitive Decline in Atrial Fibrillation - Randomized Controlled Trial (Birmingham Atrial Fibrillation Treatment of the Aged Study). Stroke. 2014;45:1381-6.

5. Baker WL, Cios DA, Sander SD, Coleman CI. Metaanalysis to assess the quality of warfarin control in atrial fibrillation patients in the United States. J Manag Care Pharm. 2009;15(3):244-52.

6. Fornari LS, Calderaro D, Nassar IB, Lauretti C, Nakamura L, Bagnatori $\mathrm{R}$ et al. Misuse of antithrombotic therapy in atrial fibrillation patients: frequent, pervasive and persistent. J Thromb Thrombolysis. 2007;23(1):65-71.

7. Lip GY, Nieuwlaat R, Pisters R, Lane DA, Crijns HJ. Refining clinical risk stratification for predicting stroke and thromboembolism in atrial fibrillation using a novel risk factor-based approach: the euro heart survey on atrial fibrillation. Chest. 2010;137(2):263-72.

8. Hachinski V, Iadecola C, Petersen RC, Breteler MM, Nyenhuis DL, Black SE et al. National Institute of Neurological Disorders and Stroke-Canadian Stroke Network Vascular Cognitive Impairment Harmonization Standards. Stroke. 2006;37(9):222041.

9. Folstein MF, Folstein SE, McHugh PR. "MiniMental State" - A practical method for grading the cognitive state of patients for the clinicians. J Psychiat Res. 1975;12:189-98.

10. Brucki SM, Nitrini R, Caramelli P, Bertolucci PH, Okamoto IH. Sugestões para utilização do MiniExame do Estado Mental no Brasil. Arq Neuropsiquiatr. 2003;61:777-81.

11. Nasreddine ZS, Phillips NA, Bédirian V, Charbonneau S, Whitehead V, Collin I et al. The Montreal Cognitive Assessment, MoCA: a brief screening tool for mild cognitive impairment. J Am Geriatr Soc. 2005;53:695-9.

12. Memória CM, Yassuda MS, Nakano EY, Forlenza OV. Brief screening for mild cognitive impairment: validation of the Brazilian version of the Montreal cognitive assessment. Int $\mathrm{J}$ Geriatr Psychiatry. 2013;28:34-40.

13. Benton A, Hamsher K. Multilingual Aphasia Examination. Iowa City: AJA Associates; 1989.

14. Machado TH, Fichman HC, Santos EL, Carvalho VA, Fialho PP, Koenig AM et al. Normative data for healthy elderly on the phonemic verbal fluency task - FAS. Dement Neuropsychol. 2009;3:55-60.

15. Wechsler D. WAIS-III Administration and Scoring Manual. New York: The Psychological Corporation, 1997. 
16. Stuss DT, Bisschop SM, Alexander MP, Levine B, Katz D, Izukawa D. The trail making test: A study in focal lesion patients. Psychol Assess 2001;13:230-9.

17. Tombaugh TN, Hubley AM. The 60-item Boston naming test: Norms for cognitively intact adults aged 25 to 88 years. J Clin Exp Neuropsychol. 1997; 19:922-32.

18. Sunderland T, Hill JL, Mellow AM, et al. Clock drawing in Alzheimer's disease. A novel measure of dementia severity. J Am Geriatr Soc. 1989;37(8):725-9.

19. Aprahamian I, Martinelli JE, Neri AL, Yassuda MS. The Clock Drawing Test A review of its accuracy in screening for dementia. Dement Neuropsychol. 2009;3(2):74-80.

20. Nitrini R, Lefèvre BH, Mathias SC, Caramelli P, Carrilho PE, Sauaia N et al. Testes neuropsicológicos de aplicação simples para o diagnóstico de demência. Arq Neuropsiquiatr. 1994;52:457-65.

21. Nitrini R, Caramelli P, Porto CS, Charchat-Fichman $\mathrm{H}$, Formigoni AP, Carthery-Goulart MT et al. Brief cognitive battery in the diagnosis of mild Alzheimer's disease in subjects with medium and high levels of education. Dement Neuropsychol. 2007;1:32-6.

22. Cummings JL, Mega M, Gray K, RosenbergThompson S, Carusi DA, Gornbein J. The neuropsychiatric inventory: comprehensive assessment of psychopathology in dementia. Neurology. 1994;44:2308-14.

23. Camozzato AL, Kochhann R, Simeoni C, Konrath CA, Pedro Franz A, Carvalho A et al. Reliability of the Brazilian Portuguese version of the Neuropsychiatric Inventory (NPI) for patients with Alzheimer's disease and their caregivers. Int Psychogeriatr. 2008;20:383-93.

24. Sheikh JI, Yesavage JA. Geriatric Depression Scale (GDS): recent evidence and development of a shorter version. Clin Gerontol. 1986;5:165-73.
25. Paradela EM, Lourenço RA, Veras RP. Validation of geriatric depression scale in a general outpatient clinic. Rev Saude Publica 2005;39:918-23.

26. Jorm AF. A short form of the Informant Questionnaire on Cognitive Decline in the Elderly (IQCODE): Development and cross-validation. Psychol Med. 1994;24:145-53.

27. Sanchez MA, Lourenço RA. Informant Questionnaire on Cognitive Decline in the Elderly (IQCODE): cross-cultural adaptation for use in Brazil. Cad Saude Publica. 2009;25:1455-65.

28. Toledo AASF, Nitrini R, Bottino CMC, Caramelli P. Brazilian research on cognitive impairment and dementia from 1999 to 2013. Dement Neuropsychol. 2014;8(4):394-8.

29. Jabos V, Cutler MJ, Day JD, Bunch TJ. Atrial fibrillation and dementia. Trends in Cardiovasc Med. 2015;25:44-51.

30. Levine DA, Galecki AT, Langa KM, Unverzagt FW, Kabeto MU, Giordani B et al. Trajectory of Cognitive Decline After Incident Stroke. J Am Med Assoc. 2015;314(1):41-51.

31. O'Brien JT, Erkinjuntti T, Reisberg B, Roman G, Sawada T, Pantoni L et al. Vascular cognitive impairment. Lancet Neurol. 2003;2:89-98.

32. Cao L, Pokorney SD, Hayden K, Welsh-Bohmer K, Newby LK. Cognitive Function: Is There More to Anticoagulation in Atrial Fibrillation Than Stroke? J Am Heart Assoc. 2015;4:e001573.

Cite this article as: Toledo AASF, Yu PC, Guallandro DM, Calderaro D, Carmo GAL, Fernandes BFS, et al. Design and protocols of the GIRAF prevention trial: A randomized, prospective, parallel, two-center study to compare cognitive outcomes in subjects with atrial fibrillation using warfarin or dabigatran. Int J Clin Trials 2015;2(4):102-7. 